\title{
Commissioning and quality assurance procedures for the HDR Valencia skin applicators
}

\author{
Domingo Granero, PhDl, Cristian Candela-Juan, PhD², Facundo Ballester, PhD³, Zoubir Ouhib, MSc'4, Javier Vijande, PhD, \\ Jose Richart, MSc ${ }^{5}$, Jose Perez-Calatayud, PhD 5,6 \\ 'Department of Radiation Physics, ERESA, Hospital General Universitario, Valencia, Spain, ${ }^{2}$ National Dosimetry Centre - CND, Instituto Nacional \\ de Gestión Sanitaria, Valencia, Spain, ${ }^{3}$ Department of Atomic, Molecular, and Nuclear Physics, University of Valencia, Burjassot, Spain, \\ ${ }^{4}$ Radiation Oncology Department, Lynn Regional Cancer Center, Boca Raton Community Hospital, Boca Raton, Florida, USA, ${ }^{5}$ Department \\ of Radiotherapy, Clínica Benidorm, Benidorm, Spain, 'DDepartment of Radiation Oncology, La Fe University Hospital, Valencia, Spain
}

\begin{abstract}
The Valencia applicators (Nucletron, an Elekta company, Elekta AB, Stockholm, Sweden) are cup-shaped tungsten applicators with a flattening filter used to collimate the radiation produced by a high-dose-rate (HDR) ${ }^{192}$ Ir source, and provide a homogeneous absorbed dose at a given depth. This beam quality provides a good option for the treatment of skin lesions at shallow depth (3-4 mm). The user must perform commissioning and periodic testing of these applicators to guarantee the proper and safe delivery of the intended absorbed dose, as recommended in the standards in radiation oncology.

In this study, based on AAPM and GEC-ESTRO guidelines for brachytherapy units and our experience, a set of tests for the commissioning and periodic testing of the Valencia applicators is proposed. These include general considerations, verification of the manufacturer documentation and physical integrity, evaluation of the source-to-indexer distance and reproducibility, setting the library plan in the treatment planning system, evaluation of flatness and symmetry, absolute output and percentage depth dose verification, independent calculation of the treatment time, and visual inspection of the applicator before each treatment. For each test, the proposed methodology, equipment, frequency, expected results, and tolerance levels (when applicable) are provided.
\end{abstract}

J Contemp Brachytherapy 20l6; 8, 5: 44l-447 DOI: $10.5114 /$ jcb.2016.63387

Key words: ${ }^{192} \mathrm{Ir}$, brachytherapy, dosimetry, Valencia applicators, commissioning, QA.

\section{Purpose}

High-dose-rate (HDR) ${ }^{192}$ Ir brachytherapy is a widely used technique in the treatment of small skin lesions [1]. Specific shielded applicators have been developed to focus the absorbed dose to the tumor volume, thus reducing absorbed dose to the surrounding healthy tissues. This is the case of the Valencia skin applicators (Nucletron, an Elekta company, Elekta AB, Stockholm, Sweden) $[2,3,4,5,6,7,8,9,10,11]$. They are tungsten cup shaped, in which the source has one single dwell position that incorporates a flattering filter providing a homogenous dose distribution at a given depth (see Figure 1).

Currently, there are two Valencia applicators commercially available. Their diameters are $2 \mathrm{~cm}$ (model $\mathrm{VH} 2$ ) and $3 \mathrm{~cm}$ (model VH3). They are designed to be used with the afterloaders microSelectron HDR Digital V3 and V2 and Flexitron (Nucletron). Each applicator is provided with two different plastic caps. One of them (not used for treatment) has a raised ring on its external surface and is used only to define the useful beam (inner applicator diameter) by pressing over the patient skin to visualize the treatment field. The other cap is smooth and is used for treatment and dosimetry purposes [12].

As for any other radiotherapy equipment, several key tests must be performed by the medical physicist prior to its clinical use. Those are: acceptance testing following the delivery of the device, thorough commissioning and implementation of a quality assurance (QA) program to evaluate periodically their functional performance characteristics, and to guarantee the proper and safe delivery of the intended absorbed dose $[1,13,14,15]$.

Recommendations on acceptance testing, commissioning, and quality control of brachytherapy equipment has been published by the Task Group 56 of the American Association of Physicists in Medicine (AAPM) [14], and by the Groupe Européen de Curiethérapie (GEC) and the European Society for Radiotherapy \& Oncology (ESTRO) [15]. Similar tests for commissioning and periodic testing used for another skin treatment device (Esteya electronic brachytherapy system from Elekta company.

Address for correspondence: Jose Perez-Calatayud, PhD, Department of Radiotherapy, Clínica Benidorm, Received: 11.05 .2016 Benidorm, Spain and Department of Radiation Oncology, La Fe University Hospital, Avda Campanar 21, Accepted: 17.09 .2016 46009 Valencia, Spain, phone: +34 96 1973329,øe-mail: perez_jos@gva.es 


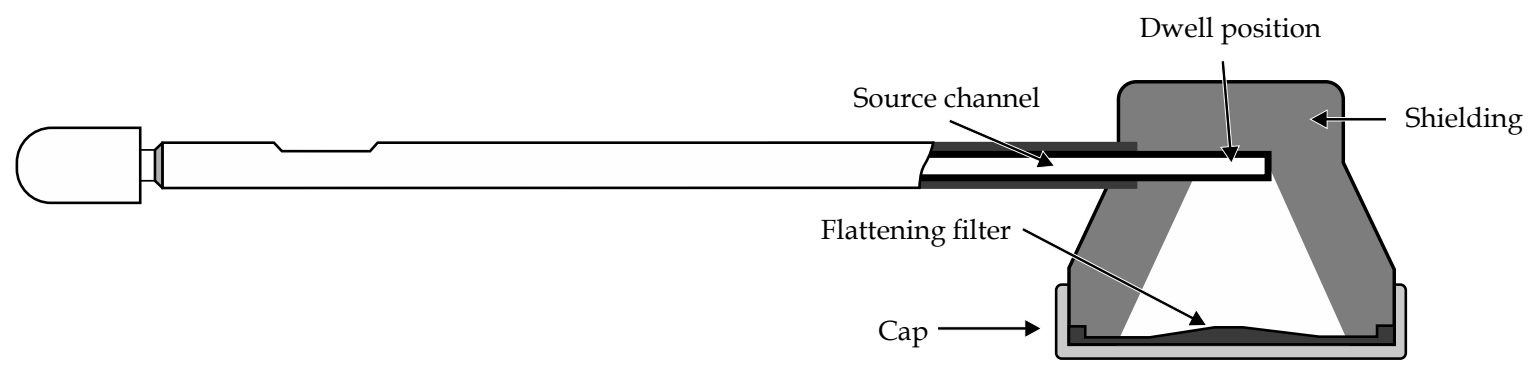

Fig. 1. Open view of the Valencia skin applicator [12]

The aim of this educational paper is to summarize in one comprehensive document various tests for commissioning and QA of the Valencia applicators that were previously published. Based on the AAPM and GEC-ESTRO guidelines for brachytherapy units as well as our experience, the methodology, equipment, frequency, expected results, and some tolerance levels are presented. We consider that including tolerance levels for all steps is out of the scope of this study, as the measurements in the $\mathrm{keV}$ energy range will be strongly dependent on the experimental methods used and on its associated uncertainties. Thus, depending on the particular measurement setup, the action levels may differ. For example, the uncertainties associated with a particular ionization chamber is different from that associated with radiochromic film measurements.

\section{Commissioning}

The following items are part of the commissioning procedure, which should be performed before the first clinical use.

\section{General considerations and verification of the manufacturer documentation and physical integrity}

The content of the user manual [12] should be fully understood, and the required internal protocols regarding the following items on the Valencia applicators should be established: intend of use, cleaning and sterilization, geometrical and dosimetric information. Each user has the responsibility to verify that all items required for the use of the Valencia applicators are accounted for and checked for any defects. This is applicable to both applicators $(2 \mathrm{~cm}$ and $3 \mathrm{~cm})$, the plastic caps, and the appropriate transfer tube.

The Valencia applicators can only be used with a specific transfer tube provided by Elekta: the GYN transfer tube no. 3 with part no. 111.004 for microSelectron HDR Digital V3 and V2 afterloader, and the GYN transfer tube no. 1 with part no. 111.681 for the Flexitron afterloader [12]. The length of the transfer tube must be verified, since it determines the source to indexer distance (SID) that is used in the treatment planning system (TPS) (see next section).

Between uses, the transfer tube must be properly stored to avoid bending it to guarantee its effective length. Before evaluating the SID value that will be used clinically, the offset between the check cable and the active source should be established. Nominally, there is a $4 \mathrm{~mm}$ (Elekta personal communication to authors) dif- ference between the length of the path of the dummy source and the length of the path of the active source, which typically is too large for the Valencia applicators given the limited length of the source channel within the applicator. If not corrected, the user will not be able to take films and readings beyond the proximal distance of the applicator filter and determine the proper SID value for each applicator. Errors due to the collision of the dummy source will be generated by the system. To avoid such errors, the user should contact the manufacturer to make these corrections.

\section{Evaluation of the source to indexer distance and reproducibility}

The Valencia treatment plan requires the SID as input to set the source at the vertex of the cone. The current nominal SID values is $1321 \mathrm{~mm}$ for the microSelectron HDR Digital V3 and V2 afterloader and $121 \mathrm{~mm}$ for the Flexitron afterloader [12]. The value of $121 \mathrm{~mm}$ for a Flexitron afterloader in combination with Valencia applicator is not the SID, but the distance from the source to the connector of the applicator. The distance from the source to the afterloader is $1121 \mathrm{~mm}$ for the Flexitron, including the length of the transfer tube of $1000 \mathrm{~mm}$. In clinical practice, these values should be measured by the medical physicist for the specific afterloader. One of the suggested methods is using radiochromic films because small SID variations have a clear influence in the symmetry of the dose distribution at the applicator exit surface. For this purpose, Lewis et al. [17] presented a practical radiochromic film dosimetry procedure. An array of ionization chambers/ diodes can also be used [18]. The latter have the practical advantage that the absorbed dose reading is obtained in real time and therefore the exit-entry transit dose can be removed. However high resolution could not be achieved due to the resolution and volume averaging effects when using an array of ionization chambers. With either radiochromic films or an array of small detectors placed in direct contact with the applicator exit surface, the dose distribution should be evaluated repeatedly while changing the SID value in 0.5 or $1 \mathrm{~mm}$ continuous steps until both the maximum and mean absorbed dose is achieved with dose distributions being symmetric (see Figure 2). In the case of the digital V3, measurements have to be collected for the 1319-1322 mm SID range in steps of $0.5 \mathrm{~mm}$ or $1 \mathrm{~mm}$ in clinical mode. However, the afterloader only allows entire values (in mm) for SID. To overcome this limitation, a combination of SID values skipping the first active position can be used. As an example, to achieve 
a measurement at dwell point $1320.5 \mathrm{~mm}$, the user will program the unit at a starting point of $1323 \mathrm{~mm}$ using a step of $2.5 \mathrm{~mm}$, and using just the second active position, which provides a measurement at $1320.5 \mathrm{~mm}$. Similar tests should be programmed in the treatment console station (TCS) to achieve measurements for all other dwell points previously mentioned. To evaluate the effect of rotation of the applicator, it is proposed to irradiate a film with the applicator rotated at $180^{\circ}$. We have done this test and the dose differences obtained with the applicator rotated $180^{\circ}$ are less than $1.5 \%$. In addition, the influence of the transfer tube bending should be evaluated, although in clinical practice it must be kept as straight as possible. The SID that results in a homogenous dose distribution will be the one used clinically as it is shown in Figure 2 (red line). Differences with respect to the nominal value greater than $1 \mathrm{~mm}$ must be investigated prior to clinical use.

\section{Setting the library plan in the TPS}

A library plan can be created on Oncentra Brachy TPS (Nucletron). It will be used to easily and safely design any patient plan. This library plan is created considering the following steps. First, one single dwell position is added at e.g., $(0,0,0) \mathrm{mm}$. Then, the SID value determined in the previous test should be introduced. After that, a dose point (the desired dose at a specific point) is defined at a distance from the dwell position and its horizontal axis of $22.39 \mathrm{~mm}$ for the $2 \mathrm{~cm}$ applicator and 26.07 for the $3 \mathrm{~cm}$ applicator. These distances are selected so that the absorbed dose (or dwell time) calculated by the TPS following the TG-43 formalism [19], equals the dose calculated at $3 \mathrm{~mm}$ depth, using Monte Carlo calculations of the Valencia applicator [2]. The dwell time corresponds to the dose prescribed by radiation oncologist and received at this point.

Some clinical situations may require the absorbed dose to be prescribed at a depth different from $3 \mathrm{~mm}$. This situation may be taken into account by means of the $F$ factor considered in the Oncentra TPS. The user may enter the value of the PDD [12] as the $F$ factor into the TPS for proper calculation of the treatment time. For example, if the prescription is done at $4 \mathrm{~mm}, F$ must be set to 0.900 (0.902) for the $2 \mathrm{~cm}(3 \mathrm{~cm})$ Valencia applicators (PDDs at $4 \mathrm{~mm}$ compared with the ones at $3 \mathrm{~mm}$ ).

To create a patient plan, the library plan created in this step can be used with the only precaution of updating the source strength, prescribed dose, $F$ factor (prescription depth), number of fractions, and starting date. It is convenient to create the patient plans using the library plan, and also in all the tests described below, in order to commission both, the applicator itself and the plan created.

\section{Evaluation of flatness and symmetry}

The dose distribution in a plane perpendicular to the axis of the radiation beam can be measured using radiochromic films. From those dose distributions, flatness and symmetry can be evaluated. This can be performed by irradiating a radiochromic film located at $3 \mathrm{~mm}$ depth (similar to common clinical prescription depth) within a solid water phantom. The use of other material as PMMA or

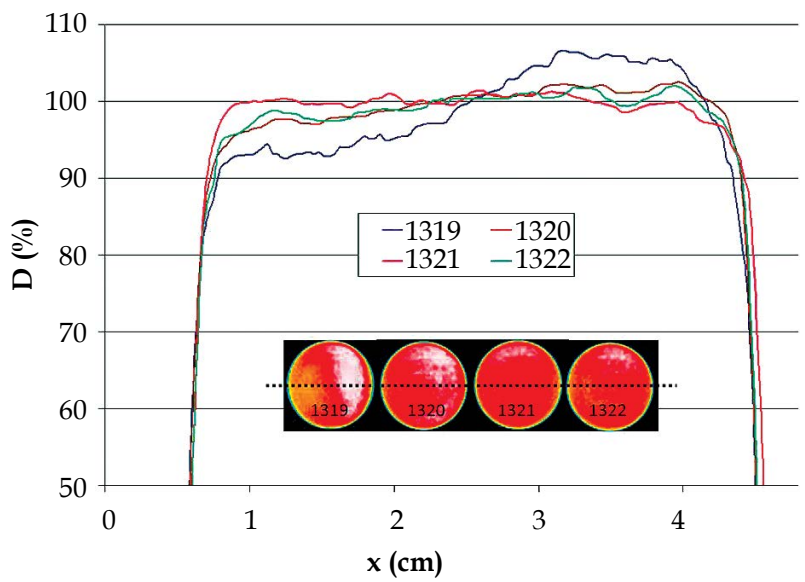

Fig. 2. Radiochromic film dosimetry of the Valencia applicators. The inset shows a 2D image of the radiochromic films for source to indexer distances (SID) of $1319 \mathrm{~mm}$, $1320 \mathrm{~mm}, 1321 \mathrm{~mm}$, and $1322 \mathrm{~mm}$. The lines show profiles (along the dotted line indicated in the inset) for each one of the SID values. The most homogenous dose distribution is associated with the SID of $1321 \mathrm{~mm}$ in this example

polystyrene is also possible for the evaluation of flatness or symmetry, although for dosimetry the preferred material is solid water. The Valencia applicator should be in full contact with the phantom surface. A plan using a dose high enough to provide an appreciable reading in the film with low noise and to neglect the possible effect of the transit dose must be created. This dose will be dependent of the type of radiochromic film. A dose of about 4 to $7 \mathrm{~Gy}$ is adequate for these films and is similar to the typical prescription dose.

The profile must be symmetric and nearly flat along the 2 or $3 \mathrm{~cm}$ circle diameter. Values of asymmetry and flatness greater than $5 \%$ should be investigated. The dose map should be compared with the Monte Carlo derived relative isodose distributions in water that can be found at www.uv.es/braphyqs.

\section{Output verification}

In this study, the term output factor (OF) refers to the dose rate per unit of air kerma strength at $3 \mathrm{~mm}$ depth on central axis. The $\mathrm{OF}$ for the Valencia applicators (in units of $\mathrm{cGy} \mathrm{h}^{-1} \mathrm{U}^{-1}$ ) should be evaluated and compared with reference data provided in Table 1, which is taken from $[2,20]$.

According to published studies [2,20], the output can be verified directly or indirectly, as described next. The direct method should be used for commissioning purposes, whereas the indirect method can be optionally used in the commissioning step in order to obtain baseline values to use for periodic quality assurance.

\section{Direct method for output measurement}

The direct method is performed by using a detector and a solid water phantom, and evaluating the dose rate. Ionization chambers are considered the gold standard for accurate clinical dosimetry [21]. However, two main dosimetric challenges arise when commissioning the HDR skin 
Table 1. Output factors for the two Valencia applicators and the mHDR-v2 source at $3 \mathrm{~mm}$ depth on the applicator central axis. The correspondence factors - $\mathrm{CF}_{\text {rev }}$ (for two different well chambers) - used for the indirect verification of the outputs are also listed. Uncertainties for output factor (OF) are for $k=1$

\begin{tabular}{lccc} 
& $\begin{array}{c}\text { OF at } 3 \mathrm{~mm} \text { depth } \\
\text { cGy h } \mathrm{h}^{-1} \mathrm{U}^{-1}\end{array}$ & \multicolumn{2}{c}{$\mathrm{CF}_{\text {rev }}$} \\
\cline { 3 - 4 } & & HDR1000 Plus & TM33004 \\
\hline VH2 & $0.225 \pm 0.005$ & 0.161 & 0.123 \\
\hline VH3 & $0.166 \pm 0.004$ & 0.180 & 0.109
\end{tabular}

applicators with these ionization chambers. First, the rapid fall off dose with depth ( $10 \%$ per mm depth) implies a high dose gradient over the measuring volume of the dosimeter, which needs to be small enough to minimize volume averaging effects. Small volume cylindrical chambers or parallel plate chamber could be used. In the former, the active diameter must be kept into the useful beam. Second, due to the fact that photons emitted by the ${ }^{192}$ Ir source have a mean energy notably lower than the ones emitted by the ${ }^{60} \mathrm{Co}$ source one used typically to calibrate the chambers, caution should be exercised with the material composition of the chambers and the energy dependence should be correctly evaluated. Consequently, the user might want to measure the OF with a suitable detector. Calibration and uncertainty issues must be carefully examined while using this method, including the active chamber size with respect to the applicator size. As commented on the literature referenced below, radiochromic film can also be used.

Pérez-Calatayud et al. [22] characterized dosimetrically the Leipzig applicators with a $0.055 \mathrm{~cm}^{3}$ Markus parallel plate ionization chamber (PTW, Freiburg, Germany), whose active volume is a cylinder $2.7 \mathrm{~mm}$ in radius and $2 \mathrm{~mm}$ in height. The chamber was calibrated with a ${ }^{60} \mathrm{Co}$ source and no energy correction factor was applied. Results were in good agreement with results from MC simulations and from TLD-100 measurements.

Granero et al. [2] commissioned experimentally the Valencia applicators with a PinPoint 31006 (PTW) ionization chamber, small TLD-100 chips, and radiochromic EBT films. Results were in agreement with those obtained from MC calculations considering the dose uncertainties of each detector. Additional details on the consistency and uncertainties of these results can be found in [2].

Usually, calibration from ${ }^{60} \mathrm{Co}$ can be used without correction factors (as in Granero et al. [2] and Pérez-Calatayud et al. [22]), although the energy dependence of other chambers not tested for ${ }^{192}$ Ir brachytherapy dosimetry should be evaluated before its application in this field. Measurements performed at our center with a new Large Field Valencia applicator without flattening filter showed discrepancies of up to $11 \%$ between measurements with the PinPoint 31006 chamber calibrated with a ${ }^{60} \mathrm{Co}$ source and the MC data. Given that this chamber has a steel electrode, its use might not be recommended in the ${ }^{192} \mathrm{Ir}$ energy range, although it provides consistent results for the Valencia applicator with flattening filter, which have higher mean photon energy. On the contrary, the newer PinPoint 31016 chamber (PTW) provides consistent results when compared to MC data for both applicators, with and without the flattening filter [23], with differences being less than $3.2 \%$ for the Valencia applicator $\mathrm{VH} 3$ when no energy correction factor is applied.

While TLD chips and films were placed at $3 \mathrm{~mm}$ depth in the cited literature, the effective point of the pin point chamber is located at $5 \mathrm{~mm}$. Therefore, when using the chamber, the reading must be translated to $3 \mathrm{~mm}$ depth by applying the PDD in order to compare the output with the data reported in Table 1.

The material, in which measurements are performed is also an important factor. Additional information regarding the validity of several solid phantom materials in $\mathrm{kV}$ (up to $280 \mathrm{kVp}$ ) dosimetry can be found in [24]. In Granero et al. [2] and Pérez-Calatayud et al. [22], polymethyl methacrylate (PMMA) phantoms (standard composition and density) were used. The correction with respect to liquid water is negligible for the ${ }^{192}$ Ir energy spectrum [21].

The measured OF must then be compared with the values listed on Table 1. If significant differences exist and are not resolved, further investigation is necessary and the applicators should not be used until there is a resolution. Discrepancies should be reported to Elekta.

Experimental uncertainties are, logically, dependent on the experimental method used. In case of using a small ionization chamber, the energy dependence with respect to the calibration quality and the effective measurement point have direct impact on the final uncertainty. However, if radiochromic film is preferred, the final uncertainty will depend on the scanning, analysis, and calibration procedures. In the following, we include some illustrative uncertainty values for some specific instrumentation.

For the case of the small Pin-Point chamber (model 31016) from PTW calibrated in ${ }^{60} \mathrm{Co}$ with the energy dependence and effective measurement point provided by the manufacturer, the output was obtained with an uncertainty of $2.6 \%(k=1)$. For radiochromic film (EBT3), calibrated for an energy of $6 \mathrm{MV}$ and scanned using the EPSON 10000XL scanner, an uncertainty of $4 \%(k=1)$ was estimated.

\section{Indirect method using the correspondence factors}

The output of the applicators can be evaluated indirectly using a well chamber and a specific insert designed to accommodate both of them. This is performed by measuring the corresponding factors (CF) $[20,25]$ and comparing them with the reference values listed in Table 1. The CFs listed here (these correspond to the revisited $C F_{\text {rev }}$ as discussed below) were obtained for a microSelectron-HDR afterloader with the mHDR-v2 source. It is noted that these $C F_{\text {rev }}$ are only valid for the exact chamber model they were measured and with the electrometer calibration factor set to 1 .

In this test, the insert is placed at the top of the well chamber and the Valencia applicator is then fitted to it without the plastic cap (Figure 3).

The two current well chambers used for these measurements are: HDR-1000-plus from Standard Imaging 

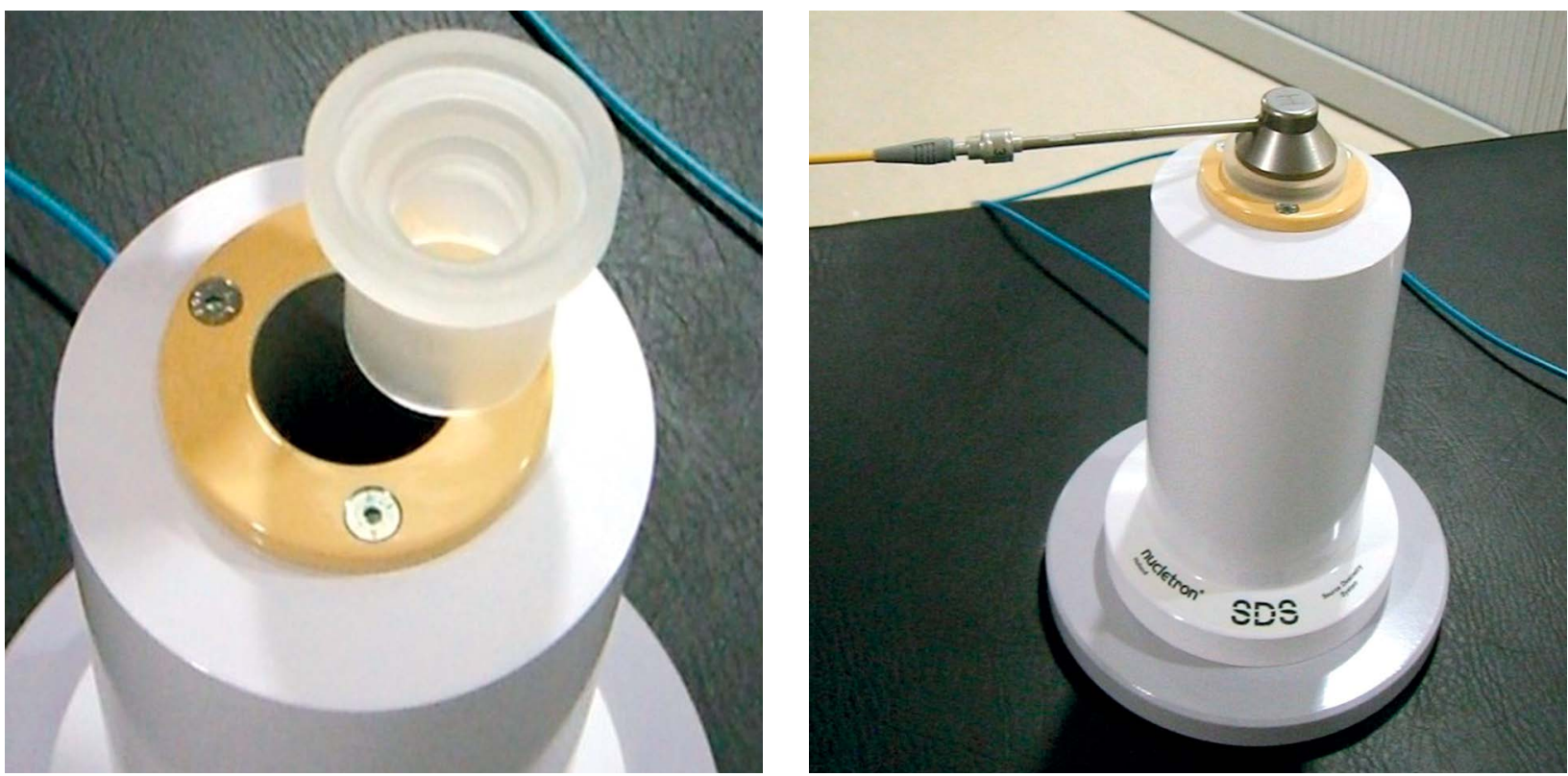

Fig. 3. Insert and set-up for the corresponding factor measurements

(Wisconsin, USA) and the TM33004 from PTW. With the specific insert from Standard Imaging, the $C F_{r e v}$ can be obtained with the following expression $[20,26,27]$ :

$$
C F_{\text {rev }}=\frac{R \times \varphi(p, \theta) \times f}{S_{K}}
$$

where $R$ is the reading collected from the well chamber and the electrometer, $\varphi(p, \theta)$ is the correction for temperature and pressure, $f$ is the calibration factor of the well chamber for the HDR source, and $S_{K}$ is the current air kerma strength of the source. Further details can be found in $[20,25]$.

In [25], Pérez-Calatayud et al. did not define properly the CF because $f$ was wrongly located in the denominator in Eq. (1). In 2008, Arthur [26] pointed out the fact that $f$ should be in the numerator such that the $C F_{\text {rev }}$ would be dimensionless. The $C F_{\text {rev }}$ was then reevaluated with the correct expression (1), and the differences between $\mathrm{CF}$ and $C F_{\text {rev }}$ were found to be small. In 2012, Gotts [27] published also that $f$ should be in the numerator being $C F_{\text {rev }}$ dimensionless. In addition, Gotts reported that the standard deviation of the calibration coefficients for HDR1000 Plus chambers calibrated at the University of Wisconsin ADCL was 2.2\%. Gotts was also critical with the application of the CF methodology questioning the assumption that the well chamber sensitivity for the external geometry (insert at the well entrance) can account for the calibration coefficient $f$, which specifies the sensitivity for the internal calibration geometry of the ionization chamber. In the reply [20], the CF promotors argued that for the three well chambers tested with the same HDR ${ }^{192}$ Ir source, the measured $C F_{\text {rev }}$ were within $1 \%$. In the reply to Gotts, $C F_{\text {rev }}$ values for both Leipzig and Valencia applicators with the correct equation were published, and are listed here for the sake of completeness in Table 1.

In the case that the $C F_{\text {rev }}$ is available, its evaluation is a convenient consistency check, always added to the direct output verification commented above. If the $C F_{\text {rev }}$ values obtained by the user differs more than $5 \%$ from the tabulated data, further investigation is needed. Such 5\% tolerance level was suggested by Pérez-Calatayud et al. [25]. In that publication, it was obtained as a combination of statistical, calibration coefficient and climatic conditions correction uncertainties. Differences obtained by clinical users have been reported. All of them were within the proposed tolerance level.

\section{Percentage depth dose curves}

The PDD for each applicator should be measured and compared with the reference data provided in Table 2 (taken from [2]). The procedure is the same as described above for the direct evaluation of the output factors. Again, an ionization chamber, TLD chips, or radiochromic films can be used [2]. However, in this case, slabs of different thicknesses are inserted between the detector and the applicator exit surface, and the detector reading is obtained as a function of depth in the phantom. The data is normalized to the reading at a depth of $3 \mathrm{~mm}$. The same plan must be irradiated for different slab thicknesses.

Differences between PDD values measured during the commissioning and PDD data listed in Table 2 should be within uncertainties (with $k=1$ ) (see e.g. [2]).

\section{Periodic testing}

This section describes the tests that we consider appropriate for a periodic QA program. With respect to the periodicity, most of the tests are to be performed when the source is replaced. After such source replacement, the most relevant elements influencing the applicator dosimetric properties are the $S_{\mathrm{K}}$ and the positioning of the source at the vertex of the applicator (SID). In principle, both elements are checked in the general test of the HDR 
Table 2. Percent depth dose (PDD) normalized at $3 \mathrm{~mm}$ on axis for the Valencia applicators $\mathrm{VH} 2$ and $\mathrm{VH} 3$

\begin{tabular}{lcc} 
& \multicolumn{2}{c}{ PDD (\%) normalized at $3 \mathrm{~mm}$ depth } \\
\cline { 2 - 3 } & Valencia H2 & Valencia H3 \\
\hline 1 & 125.6 & 125.8 \\
\hline 2 & 111.8 & 111.8 \\
\hline 3 & 100.0 & 100.0 \\
\hline 4 & 90.0 & 90.2 \\
\hline 5 & 81.3 & 81.4 \\
\hline 6 & 74.1 & 73.9 \\
\hline 7 & 67.7 & 67.6 \\
\hline 8 & 62.0 & 61.9 \\
\hline 9 & 56.8 & 56.8 \\
\hline 10 & 52.4 & 52.3 \\
\hline 15 & 36.4 & 36.6 \\
\hline 20 & 26.5 & 26.8 \\
\hline
\end{tabular}

afterloader. For the Valencia applicator output check periodicity, we suggest a 3-4 months period, simultaneously with the source replacement. In this way, it will allow double-checking, both $S_{\mathrm{K}}$ and SID.

\section{Reevaluation of the source to indexer distance, flatness and symmetry}

The SID value should be evaluated and updated after each source exchange, typically every 3 to 4 months. The main aspect that may influence the SID is the source positioning and typically it is evaluated daily for the HDR unit. The same methodology and tolerance levels, as in sub-section "Evaluation of the source to indexer distance and reproducibility", are proposed. If necessary, the SID value of the library plan should be updated.

Since the SID value might compromise the flatness and symmetry of the dose distribution at the reference depth, after each update of the library plan, the test described in sub-section "Evaluation of flatness and symmetry at $3 \mathrm{~mm}$ depth" should be repeated.

\section{Output verification at $3 \mathrm{~mm}$ depth}

After each source exchange, the output of the system must be verified following the direct or indirect method described in sub-section "Output verification". This test should make use of the library plan updated after the source replacement.

\section{Independent calculation of the treatment time}

After the treatment plan has been created with the treatment planning console (TPS), and also prior to each treatment session, the TPS planned time for that specific session should be calculated independently with, e.g., a spread sheet. An example can be found in Ref. [28]. Independent calculations can be done with the OF and PDD provided in Table 1 and Table 2, respectively, and using the actual $S_{K}$ of the source, according to:

$$
t\left(D, S_{K}, \phi, z\right)=\frac{D}{O F(\phi) \times S_{K} \times P D D(\phi, z)}
$$

where $D$ is the prescribed dose by the radiation oncologist, $\phi$ is the applicator diameter $(2 \mathrm{~cm}$ or $3 \mathrm{~cm})$, and $z$ is the prescription depth.

No differences between planned dwell times from the TPS and from the independent calculation should be found $(<1 \%)$.

\section{Safety issues when using Valencia applicators}

The cap with the ring must never be used for treatment or testing. The existing ring can introduce an air gap of roughly $1 \mathrm{~mm}$ between the applicator and the skin lesion, and therefore reduce the prescription dose by $7-10 \%$. It is also very important to verify that the cap is in place during treatment. If treatment is performed without such cap, a significant overdose $(180 \%)$ occurs due to the electron contamination component together with another overdose component due to the $1 \mathrm{~mm}$ shorter source to skin distance $(10 \%)$. This overdose has been studied by Granero et al. [29].

The cap is also used to prevent contamination between patients; these caps have to be sterilized as recommended by the manufacturer. To avoid this potential problem and considering the advantage of using just one cap for all patients (being its dosimetry impact always the same), at our department, one single non-ring or flat cap is used for all patients and dosimetry tests. To prevent the contamination between patients, the applicator is wrapped with a stressed condom.

Prior to each treatment session, the correct applicator size must be verified. This verification is important due to the size of the radiation field and the $35 \%$ output difference between the two applicators. A useful approach is to paint in different colors each applicator tip to avoid confusion.

\section{Other considerations}

In addition to those listed previously, the user is also referred to the acceptance, commissioning, and QA recommendations from societies and manufacturer regarding the HDR unit $[6,8,9]$. Furthermore, all recommendations (current and future) from the manufacturer, as well as state or federal guidelines related to the use of this device/technology should be implemented.

Although not directly as part of commissioning or QA of the Valencia applicators, adequate training should be provided and documented for the brachytherapy team (current and new members). A quality management program should be updated continuously.

\section{Conclusions}

A commissioning procedure and periodic QA program has been proposed for users of the $\mathrm{VH} 2$ and $\mathrm{VH} 3$ 
Valencia applicators, based on societal recommendations and our experience with these systems. The proposed methodology, equipment, frequency, and tolerance level (if applicable) for each test are presented. The performance of these tests guarantees proper and safe delivery of the radiation dose.

\section{Acknowledgments}

This work was supported in part by Generalitat Valenciana under Project PROMETEOII/2013/010, and by the Spanish Government under Project No. FIS2013-42156.

\section{Disclosure}

Authors report no conflict of interest.

\section{References}

1. Ouhib Z, Kasper M, Perez Calatayud J et al. Aspects of dosimetry and clinical practice of skin brachytherapy: The American Brachytherapy Society working group report. Brachytherapy 2015; 14: 840-858.

2. Granero D, Pérez-Calatayud J, Gimeno J et al. Design and evaluation of a HDR skin applicator with flattening filter. Med Phys 2008; 35: 495-503.

3. Lliso F, Granero D, Perez-Calatayud J et al. Dosimetric evaluation of internal shielding in a high dose rate skin applicator. J Contemp Brachyther 2011; 3: 32-35.

4. Granero D, Perez-Calatayud J, Ballester F et al. Radiation leakage study for the Valencia applicators. Phys Med 2013; 29: 60-64.

5. Tormo A, Celada F, Rodriguez S et al. Non-melanoma skin cancer treated with HDR Valencia applicator: clinical outcomes. J Contemp Brachyther 2014; 6: 167-172.

6. Sayler E, Eldredge-Hindy H, Dinome J et al. Clinical implementation and failure mode and effects analysis of HDR skin brachytherapy using Valencia and Leipzig surface applicators. Brachytherapy 2015; 14: 293-299.

7. Horne S. Effect of surface irregularities on dose distribution from Leipzig and Valencia HDR skin applicators. 2011. Theses and Dissertations. Paper 588.

8. Delishaj D, Laliscia C, Manfredi B et al. Non-melanoma skin cancer treated with high-dose-rate brachytherapy and Valencia applicator in elderly patients: a retrospective case series. J Contemp Brachytherapy 2015; 7: 437-444.

9. Ballester-Sánchez R, Pons-Llanas $\mathrm{O}$, Llavador-Ros $\mathrm{M}$ et al. Depth determination of skin cancers treated with superficial brachytherapy: ultrasound vs. histopathology. J Contemp Brachytherapy 2014; 6: 356-361.

10. Pons-Llanas O, Ballester-Sánchez R, Celada-Álvarez FJ et al. Clinical implementation of a new electronic brachytherapy system for skin brachytherapy. J Contemp Brachytherapy 2015; 6: $417-423$.

11. Skowronek J. Brachytherapy in the treatment of skin cancer: an overview. Postepy Dermatol Alergol 2015; 32: 362-367.

12. User Manual - Leipzig applicator set (085.040), Valencia skin applicator set (189.701), Valencia Ft skin applicator set (110.820). Nucletron. Doc. No. 777.00237MAN-05.

13. Kutcher G, Coia L, Gillin M et al. Comprehensive QA for radiation oncology: Report of AAPM radiation therapy committee task group 40. Med Phys 1994; 21: 581-618.

14. Nath R, Anderson L, Meli J et al. Code of practice for brachytherapy physics: Report of the AAPM radiation therapy committee task group No. 56. Med Phys 1997; 24: 1557-1598.
15. Venselaar J, Perez-Calatayud J (eds.). A practical guide to quality control of brachytherapy equipment, ESTRO booklet No. 8, Brussels 2004.

16. Candela-Juan C, Niatsetski Y, Ouhib Z et al. Commissioning and periodic tests of the Esteya electronic brachytherapy system. J Contemp Brachytherapy 2015; 7: 187-193.

17. Lewis D, Micke A, Yu X. An efficient protocol for radiochromic film dosimetry combining calibration and measurement in a single scan. Med Phys 2012; 39: 6339-6350.

18. Carmona V, García T, Palomo R et al. Valencia applicator commissioning using a micro-chamber array. AAPM Annual meeting - Austin. Med Phys 2014; 41: 303.

19. Rivard MJ, Coursey BM, DeWerd LA et al. Update of AAPM Task Group No. 43 Report: A revised AAPM protocol for brachytherapy dose calculations. Med Phys 2004; 31: 633-674.

20. Granero D, Perez-Calatayud J, Ballester F et al. Reply to "Comment on 'Correspondence factor for Nucletron surface applicators'". Med Phys 2012; 39: 2310-2311.

21. International Atomic Energy Agency. Absorbed dose determination in external beam radiotherapy: an international code of practice for dosimetry based on standards of absorbed dose to water. Technical Report Series No 398 (V. 12). IAEA, Vienna 2006.

22. Pérez-Calatayud J, Granero D, Ballester F et al. A dosimetric study of Leipzig applicators. Int J Radiat Oncol Biol Phys 2005; 62: 579-584.

23. Candela-Juan C, Niatsetski Y, van der Laarse R et al. Design and characterization of a new high-dose-rate brachytherapy Valencia applicator for larger skin lesions. Med Phys 2016; 43: 1639-1648.

24. Hill R, Kuncic Z, Baldock $C$. The water equivalence of solid phantoms for low energy photon beams. Med Phys 2010; 37: 4355-4363.

25. Pérez-Calatayud J, Granero D, Ballester F et al. Technique for routine output verification of Leipzig applicators with a well chamber. Med Phys 2006; 33: 16-20.

26. Arthur G. Personal Communication. Radiation Oncology Institute, Gosford 2008.

27. Gotts HS. Comment on "Correspondence factor for Nucletron surface applicators". Med Phys 2012; 39: 2947-2948.

28. Carmona V, Perez-Calatayud J, Lliso F et al. A program for the independent verification of brachytherapy planning system calculations. J Contemp Brachytherapy 2010; 2: 129-133.

29. Granero D, Candela-Juan C, Vijande J et al. Dosimetry of Leipzig and Valencia applicators without the plastic cap. Med Phys 2016; 43: 2087. 